\title{
Editorial: Pharmacokinetics and Pharmacodynamics of Pre-Exposure Prophylaxis Against HIV
}

\author{
Max von Kleist ${ }^{1,2^{*}}$, J. Gerardo García-Lerma ${ }^{3}$, Albert Liu ${ }^{4}$ and Peter L. Anderson ${ }^{5}$ \\ ${ }^{1}$ MF1 Bioinformatics, Methods Development and Research Infrastructure (MF), Robert Koch Institute, Berlin, Germany, \\ 2 Systems Pharmacology \& Disease Control, Department of Mathematics, Freie Universität Berlin, Berlin, Germany, \\ ${ }^{3}$ Laboratory Branch, Division of HIVIAIDS Prevention, National Center for HIVIAIDS, Viral Hepatitis, STD, and TB Prevention, \\ Centers for Disease Control and Prevention, Atlanta, GA, United States, ${ }^{4}$ Bridge HIV, San Francisco Department of Public \\ Health, San Francisco, CA, United States, ${ }^{5}$ Anschutz Medical Campus, University of Colorado, Denver, CO, United States
}

Keywords: PrEP, adherence, modeling, translational pharmacology, implementation

Editorial on the Research Topic

Pharmacokinetics and Pharmacodynamics of Pre-Exposure Prophylaxis Against HIV

\section{BACKGROUND}

In 2018 about 1.7 million individuals became infected with the human immunodeficiency virus $(\mathrm{HIV})^{1}$. While therapies are highly effective in suppressing virus replication and reducing transmission, viral rebound generally occurs within weeks after treatment discontinuation (Chun

OPEN ACCESS

Edited and reviewed by:

Alastair George Stewart,

The University of Melbourne, Australia

${ }^{*}$ Correspondence: Max von Kleist kleistm@rki.de

Specialty section: This article was submitted to Translational Pharmacology, a section of the journal

Frontiers in Pharmacology

Received: 09 July 2020 Accepted: 04 August 2020 Published: 26 August 2020

Citation: von Kleist M, García-Lerma JG, Liu A and Anderson PL (2020)

Editorial: Pharmacokinetics and Pharmacodynamics of Pre-Exposure Prophylaxis Against HIV.

Front. Pharmacol. 11:1288. doi: 10.3389/fphar.2020.01288 et al., 2015). The establishment of a latent virus reservoir early in infection poses challenges for identifying effective HIV cure strategies. Vaccines have had limited success to date (Rerks-Ngarm et al., 2009; Caskey et al., 2019) although some promising strategies are under evaluation. While a major success in HIV research has been the development of highly effective antiretrovirals, a fruitful idea is to re-purpose those drugs for HIV prevention. Substantial progress has been made developing antiretroviral (ARV)-based strategies to prevent HIV transmission, including preexposure prophylaxis (PrEP) (Grant et al., 2010). PrEP with oral FTC in combination with tenofovir disoproxil fumarate (TDF) or tenofovir alafenamide (TAF) is an established prevention strategy to protect certain populations at risk of HIV acquisition.

By inhibiting early virus replication, PrEP drugs increase the chance of virus elimination before a new host is irreversibly infected. However, viral inhibition-and thus PrEP efficacy-largely depends on the drugs' concentration at the target site. While initial PrEP trials with once daily oral FTC/TDF estimated a moderate efficacy based on an intent-to-treat analysis (see ${ }^{2}$ for an overview), subsequent analyses indicated that if individuals adhere to the once-daily regimen, protection levels of $80-99 \%$ may be reached (Grant et al., 2014). These analyses revealed a certain level of pharmacologic forgiveness with variable adherence, which was evident in the IPERGAY trial that showed high efficacy for evident-driven dosing (Antoni et al., 2020).

This Research Topic compiles articles addressing pharmacokinetic/pharmacodynamic (PK/PD) aspects of PrEP. A particular focus is on PrEP adherence, on translational research to predict PrEP efficacy, as well as innovative approaches to dispensing clinical trial PrEP drugs.

\footnotetext{
${ }^{1}$ UNAIDS: FACT SHEET - GLOBAL AIDS UPDATE 2019 (https://www.unaids.org/en/resources/fact-sheet).

${ }^{2}$ Global Advocacy for HIV prevention, https://www.avac.org/sites/default/files/u3/By_The_Numbers_PrEP.pdf.
} 


\section{PREP ADHERENCE}

Mallayasamy et al. analyzed data from 920 individuals to identify demographic, as well as socio-behavioral factors associated with PrEP adherence in sero-discordant couples in East Africa. They found that older age, female gender, and sexual activity were associated with increased adherence to FTC/TDF, whereas having a partner on ART $>6$ months, being in the study for $>6$ months, and problematic alcohol use were associated with lower adherence. These data were gathered objectively using electronic adherence monitoring systems, which may not be available in other PrEP implementation studies and broader roll-out. How can adherence be assessed in real-world settings to interpret and analyze PrEP outcomes? Blumenthal et al. investigated, in a group of HIV-uninfected men who have sex with men (MSM) from the TAPIR study, which self-reported adherence questions correlate with objective measures of drug adherence, as measured by tenofovir diphosphate (TFV-DP) concentrations in dried blood spots (DBS). They found that answers to the question "Thinking about the past 4 weeks, what percentage of the time were you able to take all your PrEP medications" were most strongly associated with objective adherence measurements in this cohort. Pyra et al. analyzed whether TFV-DP levels in DBS, which can be conveniently collected and stored, correlate well with the history of drug intake measured by electronic adherence systems. Lalley-Chareczko et al. analyzed whether tenofovir (TFV) levels in urine after administration of TAF may be a good indicator of recent drug adherence. TAF is a tenofovir prodrug approved for PrEP in combination with FTC for high-risk men and transgender women who have sex with men. Lalley-Chareczko et al. found that urine levels of TFV persist at detectable concentrations in participants taking TAF for at least 7 days despite largely undetectable plasma TFV concentrations, suggesting that urine may be a good marker for recent drug adherence.

\section{TRANSLATIONAL PREP RESEARCH}

Quantifying PrEP efficacy from clinical data requires determining the relative rate of seroconversion in the intervention $v s$. control arm. A major statistical challenge arises from the fact that sexual HIV transmission probabilities are extremely low (Royce et al., 1997) and hence the number of seroconversions in a clinical trial, which is used to calculate efficacy is prone to statistical errors (Dunn and Glidden, 2016). Consequently, PrEP development requires making use of the entire translational research toolbox. Herrera discussed the toolbox of in vitro and ex vivo models used to screen PrEP compounds, characterize their pharmacology, evaluate their safety, and determine target drug levels. Additionally, computational modeling approaches offer flexible and powerful tools to study drug behavior, integrate different data sources, and predict clinical endpoints. Duwal et al. used an integrated pharmacokinetic-viral dynamic computer model
(Duwal et al., 2018). They first developed a pharmacokinetic (PK) model for efavirenz based on in vitro and clinical data from the ENCORE-1 study. Subsequently, they integrate this PK model with a stochastic virus response model to predict the prophylactic efficacy of efavirenz following different dosing schemes. Based on these simulations, they propose that lowdose efavirenz could have high clinical efficacy as PrEP. Straubinger et al. give an overview of mathematical modeling efforts. They summarize approaches for approved and currently developed PrEP drugs from a PK/PD perspective, as well as approaches that connect pharmacology and viral dynamics to ultimately predict PrEP efficacy in relation to drug dosing and provide a short outlook on epidemiological modeling of PrEP efficacy.

\section{TRIAL DESIGN}

Lastly, Lal et al. studied in Australia, whether community based pharmacies, which offer more convenience for study participants, can undergo training and modifications to achieve good clinical practice compliance to dispense clinical trial study drugs. Overall, they recorded very few deviations from study protocols, indicating that community-based pharmacies should be considered in HIV prevention trials.

\section{CONCLUSION}

PrEP is a very active field of investigation at the intersection of pharmacology, behavior, and public health. When taken as prescribed, oral PrEP is highly effective although inadequate adherence reduces efficacy and public health benefit. The current issue provides a snapshot of current research activities with a focus on PK/PD aspects. As the PrEP field continues to evolve, interest is now shifting to long-acting drug formulations and sustained drug delivery systems that can overcome some of the adherence issues associated with daily PrEP.

\section{AUTHOR CONTRIBUTIONS}

All authors listed have made a substantial, direct, and intellectual contribution to the work, and approved it for publication.

\section{FUNDING}

MK acknowledges support from the BMBF projects "Meth4SysPharm," as well as "Trans-PrEP" (grant numbers 031A307, 01KI2016). AL is supported by several grants from the National Institutes of Health and the California HIV Research Program. He has also received research funding from Gilead Sciences and Viiv Healthcare to conduct investigator sponsored 
research and has led studies in which Gilead had donated study drug. He has also received support from IAS-USA for manuscript preparation and Practice Point Communications for conducting continuing education activities. PA is supported by $\mathrm{NIH}$ R01 AI122298.

\section{REFERENCES}

Antoni, G., Tremblay, C., Delaugerre, C., Charreau, I., Cua, E., Rojas Castro, D., et al. (2020). On-demand pre-exposure prophylaxis with tenofovir disoproxil fumarate plus emtricitabine among men who have sex with men with less frequent sexual intercourse: a post-hoc analysis of the ANRS IPERGAY trial. Lancet HIV 7 (2), e113-e120. doi: 10.1016/S2352-3018(19)30341-8

Caskey, M., Klein, F., and Nussenzweig, M. C. (2019). Broadly neutralizing antiHIV-1 monoclonal antibodies in the clinic. Nat. Med. 25 (4), 547-553. doi: 10.1038/s41591-019-0412-8

Chun, T.-W., Moir, S., and Fauci, A. S. (2015). HIV reservoirs as obstacles and opportunities for an HIV cure. Nat. Immunol. 16 (6), 584-589. doi: 10.1038/ni.3152

Dunn, D. T., and Glidden, D. V. (2016). Statistical issues in trials of preexposure prophylaxis. Curr. Opin. HIV AIDS 11 (1), 116-121. doi: 10.1097/ COH.0000000000000218

Duwal, S., Dickinson, L., Khoo, S., and von Kleist, M. (2018). Hybrid stochastic framework predicts efficacy of prophylaxis against HIV: An example with different dolutegravir prophylaxis schemes. PloS Comput. Biol. 14 (6), e1006155. doi: 10.1371/journal.pcbi.1006155

Grant, R. M., Lama, J. R., Anderson, P. L., McMahan, V., Liu, A. Y., Vargas, L., et al. (2010). Preexposure chemoprophylaxis for HIV prevention in men who have sex with men. $N$ Engl. J. Med. 363 (27), 2587-2599. doi: 10.1056/ NEJMoa1011205

Grant, R. M., Anderson, P. L., McMahan, V., Liu, A., Amico, K. R., Mehrotra, M., et al. (2014). Uptake of pre-exposure prophylaxis, sexual practices, and HIV incidence in men and transgender women who have sex with men: a cohort study. Lancet Infect. Dis. 14 (9), 820-829. doi: 10.1016/S1473-3099(14)70847-3

\section{ACKNOWLEDGMENTS}

We would like to thank the Frontiers editorial staff, all the authors who contributed excellent papers, and the reviewers whose work has made publication of this Research Topic possible.

Rerks-Ngarm, S., Pitisuttithum, P., Nitayaphan, S., Kaewkungwal, J., Chiu, J., Paris, R., et al. (2009). Vaccination with ALVAC and AIDSVAX to prevent HIV-1 infection in Thailand. N Engl. J. Med. 361 (23), 2209-2220. doi: 10.1056/ NEJMoa0908492

Royce, R. A., Seña, A., Cates, W. Jr, and Cohen, M. S. (1997). Sexual transmission of HIV. N Engl. J. Med. 336 (15), 1072-1078. doi: 10.1056/NEJM199704 103361507

Conflict of Interest: GG-L is named in US Government patents on "Inhibition of HIV infection through chemoprophylaxis" and in US Government patent applications on "HIV post-exposure prophylaxis" and "HIV pre-exposure prophylaxis." The findings and conclusions of this manuscript are those of the authors and do not necessarily represent the official views of the Centers for Disease Control and Prevention. AL has received research funding from Gilead Sciences and Viiv Healthcare to conduct investigator sponsored research and has led studies in which Gilead had donated study drug. PA has received personal fees and research funding from Gilead Sciences.

The remaining author declares that the research was conducted in the absence of any commercial or financial relationships that could be construed as a potential conflict of interest.

Copyright (c) 2020 von Kleist, Garcia-Lerma, Liu and Anderson. This is an open-access article distributed under the terms of the Creative Commons Attribution License (CC BY) The use, distribution or reproduction in other forums is permitted, provided the original author(s) and the copyright owner(s) are credited and that the original publication in this journal is cited, in accordance with accepted academic practice. No use, distribution or reproduction is permitted which does not comply with these terms. 